based upon the tREACH randomization within each stratum (figure 1): $[\mathrm{A}]$ triple DMARD combination therapy (MTX $25 \mathrm{mg} / \mathrm{wk}$.+SASP $2 \mathrm{gr} / \mathrm{day}+\mathrm{HCQ} 400 \mathrm{mg} /$ day+GCs (intramuscular or an oral tapering scheme, starting with $15 \mathrm{mg} /$ day), [B] MTX $25 \mathrm{mg} / \mathrm{wk} . \pm \mathrm{GCs}$ orally starting with $15 \mathrm{mg} /$ day, [C] HCQ $400 \mathrm{mg} /$ day and [D] GCs orally starting with $15 \mathrm{mg} /$ day. Treatment strategies were 'tightly controlled', with patients being examined every 3 months. Treatment decisions were based upon the original Disease Activity Score (DAS) threshold for low disease activity (DAS <2.4). Primary outcomes were DAS and functional ability, measured with the Health Assessment Questionnaire (HAQ), over time, using a linear mixed model (LMM). In our final model we corrected for baseline DAS and HAQ and visser score, which is a confounder by indication on forehand.

Results: 162 patients were grouped into treatment arms A ( $n=17), B(n=64), C$ $(n=40)$ or $D(n=41)$. Patients were mostly female $(67 \%)$ with an average symptom duration of 161 days (95\% Cl: 146-175). At baseline the average visser score was 4 out of 13 (IQR 4-5). The difference in visser score was mainly due to the difference in erosions (figure $1 \mathrm{~A}$ ). Figure $1 \mathrm{~B}$ and $\mathrm{C}$ show the DAS and $\mathrm{HAQ}$ over time per treatment arm. Our corrected LMM showed that there was no significant difference between treatment arms for DAS over time. After 3 months $56 \%, 38 \%$, $35 \%$ and $69 \%$ respectively treated with $A, B, C$ and D had an active disease (DAS $\geq 2.4$ ), and thus needed a treatment intensification ( $P 0.003$ for $C$ versus $D$ ). However, after 1 year there was no difference between DAS over all treatment arms. HAQ over time did differ between treatment arms. Patients who received HCQ showed a better functional ability over time compared to patients receiving 1 of the other treatments $(C$ versus respectively $B(\beta=-0.18, p 0.000), D(\beta=-0.14$, $\mathrm{p} 0.004)$ and $A(\beta=-0.17, \mathrm{p} 0.016))$.

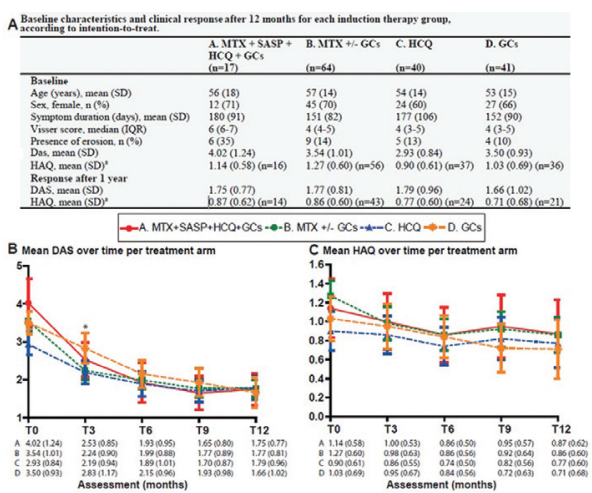

Abstract SAT0078 - Figure 1. (A) Baseline characteristics and clinical response after 12 months for each induction therapy group, according to intention-to-treat. (B) Mean DAS over time per treatment arm. (C) Mean HAQ over time per treatment arm. ${ }^{a}$ Not everyone filled out a (complete) questionnaire and therefore $\mathrm{n}$ is different for HAQ. MTX $25 \mathrm{mg} / \mathrm{wk}$, SASP 2 $\mathrm{gr} /$ day, HCQ $400 \mathrm{mg} /$ day, GCs intramuscular or an oral tapering scheme starting with $15 \mathrm{mg} /$ day for treatment $A$ and only oral for treatment $B+D$. * $P 0.011$ for $C$ versus $D$. Abbreviations: DAS, Disease Activity Score; GCs, glucocorticoids; HAQ, Health Assessment Questionnaire; HCQ, hydroxychloroquine; MTX, methotrexate; RA, rheumatoid arthritis; SASP, sulfasalazine

Conclusions: This research supports current hypothesis that seronegative RA patients can be treated with less aggressive treatment with similar efficacy. Disclosure of Interest: None declared

DOI: 10.1136/annrheumdis-2018-eular.3214

\section{SAT0079 TREATMENT EXPECTATIONS INFLUENCE BOTH SUBJECTIVE AND OBJECTIVE OUTCOME PARAMETERS IN PATIENTS WITH RHEUMATOID ARTHRITIS- A PROSPECTIVE COHORT STUDY}

J. Mucke $^{1}$, R. Brinks ${ }^{1}$, A. Dimitriou ${ }^{2}$, J. Richter ${ }^{1}$, M. Schneider ${ }^{1} .{ }^{1}$ Policlinic for Rheumatology and Hiller-Research Centre for Rheumatology, Heinrich-HeineUniversity Duesseldorf, Düsseldorf, Germany; ${ }^{2}$ Department of Rheumatology, Stadtspital Triemli, Zurich, Switzerland

Background: The prediction of individual response to treatment in rheumatoid arthritis (RA) is challenging and often limited. Here we evaluate the influence of patients' expectations and attitudes towards newly initiated disease-modifying anti-rheumatic drugs (DMARDs) on clinical outcome in RA.

Methods: 100 patients (74 female) with RA according to 2010 ACR/EULAR classification criteria with upcoming change in DMARD treatment were included. Patients' treatment beliefs, health related quality of life, treatment expectations, and painrelated cognitions were measured using the beliefs about medicines questionnaire (BMQ), the SF-36, the questionnaire about patient expectation (PE), and the pain-related self-statement scale (PRSS), respectively before treatment initiation (T0) and their DAS28-CRP was calculated at T0 and after 4 months (T4). Associations between patients' beliefs, expectations and their attitude according to the questionnaires and changes in DAS28-CRP between T0 and T4 were explored by regression analyses using the Aikaike information criterion.

Results: Regression analyses revealed that $42.2 \%$ of all variability in treatment response measured as a decline in DAS28-CRP ( $\triangle \mathrm{DAS} 28$ ) could be explained by expectations, psychological factors and laboratory parameters assessed with the applied questionnaires. Among these we identified the expected improvement rate with $23.4 \%$ as well as the patients' fear of side effects with $22.0 \%$ as the main predictors of $\triangle \mathrm{DAS} 28$. The CRP-value at T0 accounted with $15 \%$ to the variability in $\triangle$ DAS28. Other highly influential factors were PRSS catastrophizing scale $(10.7 \%)$, the BMQ concern scale $(8.1 \%)$, other BMQ scales $(7.9 \%)$ and medications' route of administration (8.0\%).

Conclusions: The present study indicates a high impact of patients' expectations and their attitude towards new therapies on clinical response effecting both objective and subjective outcome parameters. Integration of individual patient's preferences and their expectations in treatment decisions and management can significantly increase treatment response.

Disclosure of Interest: None declared

DOI: 10.1136/annrheumdis-2018-eular.4847

\section{SAT0080 SECULAR TRENDS PRIOR TO-AND AFTER DISSEMINATION OF BEST PRACTICE RECOMMENDATIONS SHOWED EARLIER INTENSIFIED MEDICATION STRATEGIES AND IMPROVED OUTCOMES IN CANADIANS WITH EARLY INFLAMMATORY ARTHRITIS}

O. Schieir ${ }^{1}$, S.J. Bartlett ${ }^{2}$, M.-F. Valois ${ }^{3}$, J.E. Pope ${ }^{4}$, C. Thorne ${ }^{5}$, C. Hitchon ${ }^{6}$, B. Haraoui ${ }^{7}$, G. Boire ${ }^{8}$, D. Tin ${ }^{5}$, E.C. Keystone ${ }^{9}$, V.P. Bykerk ${ }^{9,10}$ on behalf of on behalf of the CATCH Investigators. ${ }^{1} \mathrm{U}$ Toronto, Toronto; ${ }^{2}$ Epidemiology, ${ }^{3} \mathrm{McGill}$ University, Montreal; ${ }^{4} \mathrm{U}$ Western Ontario, London; ${ }^{5}$ Southlake Regional Health Center, Newmarket; ${ }^{6} \cup$ Winnipeg, Winnipeg; ${ }^{7}$ Institute de Rhumatologie, Montreal; ${ }^{8} \mathrm{U}$ Sherbrooke, Sherbrooke; ${ }^{9}$ Rheumatology, U Toronto, Toronto, Canada;

${ }^{10}$ Rheumatology, Hospital for Special Surgery, New York, USA

Background: International and local best-practice recommendations released in 2010-11 aimed to improve outcomes in early RA via earlier diagnosis and a treatto-target approach. These should be associated with improved outcomes.

Objectives: To examine secular trends in patient characteristics and treatment strategies at RA diagnosis and disease activity outcomes in the 1 st year of follow-up comparing earlier (2007-2010) and later (2011-2016) time periods, prior to and following dissemination of 2011 guidelines in a large Canadian early inflammatory arthritis (EIA) cohort of RA patients.

Methods: Data were from patients with early classifiable $(87 \%)$ or probable (13\%) RA (<1 year of symptoms) enrolled each year in an ongoing prospective multi-centre cohort study between 2007-2016 with $\geq 12 \mathrm{M}$ follow-up undergoing 3-monthly visits including clinical assessments, questionnaires, and laboratory tests in the 1 st year. Treatment was decided by their rheumatologist. These were cohort investigators who met annually to discuss means to improve outcomes. Descriptive statistics compared patient characteristics, early treatment strategies with conventional synthetic(cs) and biologic(b) DMARDs and disease activity (DAS28) outcomes. Differences in DAS28 targets achieved per time period were compared using Chi-Squares and medication doses by Cochrane Hermitage trend tests.

Results: Of 2227 patients enrolled in CATCH (Canadian Early Arthritis Cohort), symptom duration was 6 (3) months. There was a slight increase in number recruited, education and income and slight decrease in baseline symptom duration from early to later time periods. Baseline smoking, obesity rates, comorbidities, positive serology, inflammatory markers and joint counts did not differ significantly between time periods. Baseline erosions were less frequent (17\% vs. $24 \%, \mathrm{p}<0.0001$ ) and mean symptom duration decreased slightly (5.6 vs. 5.9 months, $\mathrm{p}=0.018)$ in earlier vs later periods. Most $(87 \%)$ entered in moderate or high disease activity (MDA or HDA);disease activity at 3, 6 and $12 \mathrm{M}$ markedly improved over calendar time (figure 1). Respective DAS28 REM/LDA rates at 12 $M$ from early to late periods significantly increased $(p<0.01), 20 \%(p<0.001)$ used higher doses of MTX ( $\geq 20 \mathrm{mg}$ ); and more used subcutaneous (sc) MTX, MTX combo therapies sooner and more rapidly escalation to bDMARDs all p's $<0.05$ ). 